\title{
STUDY ON THE PROUDMAN RESONANCE OF WAVES INDUCED BY A MOVING ATMOSPHERIC PRESSURE DISTURBANCE
}

\author{
Xiaojing Niu, Tsinghua University,nxj@tsinghua.edu.cn \\ Yixiang Chen, Tsinghua University, c-yx16@mails.tsinghua.edu.cn \\ Haojie Zhou, Tsinghua University, zhou-hj14@mails.tsinghua.edu.cn
}

\section{INTRODUCTION}

A moving atmospheric pressure disturbance can induce a system of forced water waves. As predicted by the linear theory, an infinite wave height will be induced when the Froude number $\mathrm{Fr}=1$, which is known as the Proudman resonance. Fr is defined as the ratio between the moving speed of an atmospheric pressure disturbance and the phase velocity of shallow water wave. The Proudman resonance is thought to be one of main mechanisms for the destructive meteotsunami (Monserrat et al., 2006). In this study, the nonlinear shallow water equations are used to describe the waves induced by a moving pressure disturbance, and the impact factors to the maximum water elevation in the case of $\mathrm{Fr}=1$ are discussed.

\section{THE RESONANCE PHENOMENON}

The forced wave induced by a low pressure of centrosymmetric distribution, which is travelling with a constant velocity in an unbounded sea of constant depth, has been numerically investigated (Niu and Zhou, 2015). When Fr<1, as shown in Fig 1(a), the distribution of water surface elevation is similar to the pressure disturbance, and the maximum water elevation is always larger than that can be induced by a static low pressure. When Fr>1, as shown in Fig 1(c), a triangle shaped wave pattern is formed with a depression occurs in front of the pressure center, and the maximum water elevation can be even smaller than that in the case of a static low pressure. $\mathrm{Fr}=1$ is a critical point that the wave pattern significantly changes and the maximum water elevation is obviously larger than other cases, as shown in Fig1 (b),

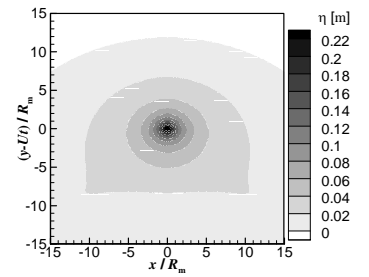

(a) $\mathrm{Fr}=0.5$

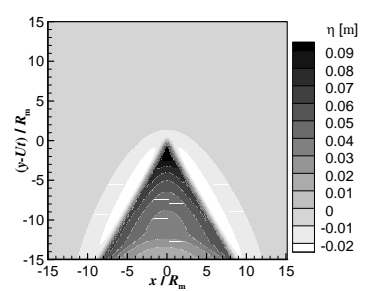

(c) $\mathrm{Fr}=2.0$

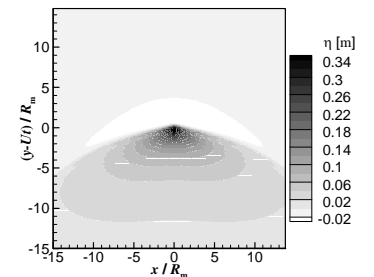

(b) $\mathrm{Fr}=1.0$

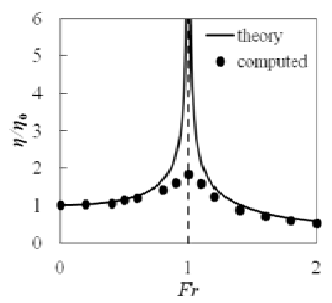

(d) maximum elevation
Figure 1 - Water surface elevation induced by a moving low pressure

As Proudman's linear theory suggested, Fr acts as the key parameter which affects the wave height. As widely known, a decrease of atmospheric pressure with $1 \mathrm{hPa}$ will lead to $1 \mathrm{~cm}$ elevation of water level under the static condition. The maximum water elevation under the static condition $\eta_{0}$ is introduced as a reference. It can be seen that the relative maximum water elevation $\eta / \eta_{0}$ increases with $\mathrm{Fr}$ when $\mathrm{Fr}<1$ and then decreases when $\mathrm{Fr}>1$, as shown in Fig 1(d). The water elevation can be significantly larger in the case of a pressure disturbance moving with a speed similar to the wave velocity. An infinite wave height is predicted by the linear theory at $\mathrm{Fr}=1$. But in practice and as predicted by the nonlinear shallow water model, the maximum water elevation in the case of $\mathrm{Fr}=1$ is finite and its value depends on many factors.

\section{ACCUMULATION OF WAVE ENERGY}

The evolution of the wave in case of $\mathrm{Fr}=1$ is numerically investigated. A free wave is separated from the forced wave and moving backward. Based on the numerical result, the forced wave can reach a relatively stable stage after the pressure disturbance travels for a long time. The variation of kinetic energy and potential energy is computed, and the energy transfer from the pressure disturbance to water is discussed.

\section{CONTRIBUTIONS OF EACH TERM IN SHALLOW WATER EQUATION}

The stable water elevation at $\mathrm{Fr}=1$ is related to flow nonlinearity, bottom friction, water viscosity and so on. These impact factors are systematically evaluated by switching on/off the corresponding item or adjusting the coefficient in the numerical model. The impacts on the water elevation of each term in a specific case are computed. The results show that the bottom friction and the nonlinearity have larger impacts and the impact of the viscosity can be neglected. The contributions of each term vary with water depth and the parameters of pressure disturbances.

\section{INFLUENCES OF DISTURBANCE PARAMETERS}

Based on the numerical simulation and theoretical analysis, the impacts of the disturbance parameters on the maximum water elevation in the case of $\mathrm{Fr}=1$ are further discussed. The maximum of water surface elevation is approximately in proportion to the central pressure drop, and slightly affected by the spatial scale of pressure disturbance. A pressure disturbance with smaller spatial scale and smaller central pressure drop will give a larger $\eta / \eta_{0}$.

\section{REFERENCES}

Monserrat, S., Vilibić, I. and Rabinovich, A.B., (2006). Meteotsunamis: Atmospherically induced destructive ocean waves in the tsunami frequency band. Natural Hazards and Earth System Sciences, 6(6): 1035-1051. Niu, X. and Zhou, H., (2015). Wave pattern induced by a moving atmospheric pressure disturbance. Applied Ocean Research, 52: 37-42. 